\title{
Entrapment of the Fifth Lumbar Spinal Nerve by Advanced Osteophytic Changes of the Lumbosacral Zygapophyseal Joint: A Case Report
}

\author{
Yu-ichiro Ohnishi, ${ }^{1,2}$, Takamichi Yuguchi ${ }^{2}$, Koichi Iwatsuki $^{1}$, Toshiki Yoshimine ${ }^{1}$ \\ ${ }^{1}$ Department of Neurosurgery, Osaka University School of Medicine, Suita, Osaka, Japan \\ ${ }^{2}$ Spine and Spinal Cord Center, Hanwa Memorial Hospital, Osaka, Japan
}

\begin{abstract}
A 54-year-old female patient had a 6-year history of backache and left sciatica. Five years earlier, she had undergone surgery in another hospital for left L4-5 disc herniation. Computed tomography revealed the ossified wall that enclosed the left L5 nerve root. There were also osteophytic changes in the left L5-S zygapophyseal joint. These osteophytes developed rostrally, along the left L5 nerve root, through the intervertebral foramina. We performed decompression surgery for the left L5 nerve root, and surgery resulted in symptomatic relief. We experienced a rare clinical presentation of osteophytic formation, with a specific configuration in relation to the nerve root. Surgeons should be aware of entrapment of the lumbar spinal nerve by advanced osteophytic changes occurring in the zygapophyseal joint after lumbar surgery.
\end{abstract}

Key Words: Osteophyte, Entrapment, Nerve root, Zygapophyseal joint

\section{Introduction}

Advanced degenerative changes in the zygapophyseal joints are of essential clinical interest, because the intervertebral foramina obstructed by these processes may cause severe neurological symptoms. This study is a case report demonstrating a rare clinical presentation of osteophytic formation, with a specific configuration in relation to the nerve root.

\section{Case Report}

\section{History}

A 54-year-old female patient had a 6-year history of backache and left sciatica. Five years earlier, she had undergone surgery in another hospital for L4-5 disc herniation. Although her left sciatica subsided, she experienced gradual deterioration of symptoms, including pain, numbness, and weakness in the left leg.

\section{Examinations}

On referral to our hospital, the patient's neurological examination showed left L5 radiculopathy. The strengths of dorsiflexion were weak at the ankle and great toe. Light touch sensation was decreased over the dorsum of the left foot and pain was present. The left patellar reflex was decreased, but the Babinski sign was absent. Routine laboratory tests were normal and the patient was not diabetic. On magnetic resonance imaging, there was slight L4-5 disc bulging, but no canal stenosis. Computed tomography (CT) revealed that ossified wall enclosed the left L5 nerve root at the lateral recess (Fig. 1A, B). Sagittal and coronal views also showed ossified wall medially located from the left L5 pedicle (Fig. 1C, D). There were also osteophytic changes

Received Jun 27, 2011; Revised Jul 8, 2011; Accepted Jul 25, 2011

Corresponding author: Yu-ichiro Ohnishi, MD

Department of Neurosurgery, Osaka University School of Medicine,

2-2 Yamadaoka, Suita, Osaka 565-0871, Japan

Tel: +81-6-6879-3652, Fax: +81-6-6879-3659, E-mail: ohnishi@nsurg.med.osaka-u.ac.jp 
in the left L5-S zygapophyseal joint. Left L4-5 partial laminectomy and left L4-5 partial facetectomy had been performed at previous hospital. Osteophytes originating from the left L5-S zygapophyseal joint developed rostrally, along the left L5 nerve root (Fig. 1).

\section{Operation and postoperative course}

The imaging findings could explain the left L5 radiculopathy. It is suggested that abnormal ossification of the left L5 lateral recess derived from the osteophytes in the left L5-S zygapophyseal joint. Left L4-5 unilateral laminectomy and partial facetectomy had been performed and the shoulder of the left L5 nerve root was confirmed. The distal segment of the left L5 nerve root was embedded with abnormal ossified formation that corresponded on $\mathrm{CT}$ to the ossified wall at the left lateral recess of L5. We drilled this abnormal ossification to expose the left L5 nerve root (Fig. 2A). This ossification continued to the superior left L5 articular process and the distal part of the intervertebral foramina. We carefully decompressed the nerve root from the left lateral recess to the intervertebral foramina (Fig. 2B). The fat tissue in the epiradicular sheath disappeared through the intervertebral foramina. Unfortunately, we could not perform histological examination of these ossified tissues. The lateral half of the left L4-5 joint was preserved. Postoperative CT showed removal of the ossified lesion. After surgery, the patient's neurological function improved, and pain in her left leg subsided. Postoperative external fixation continued for three months.
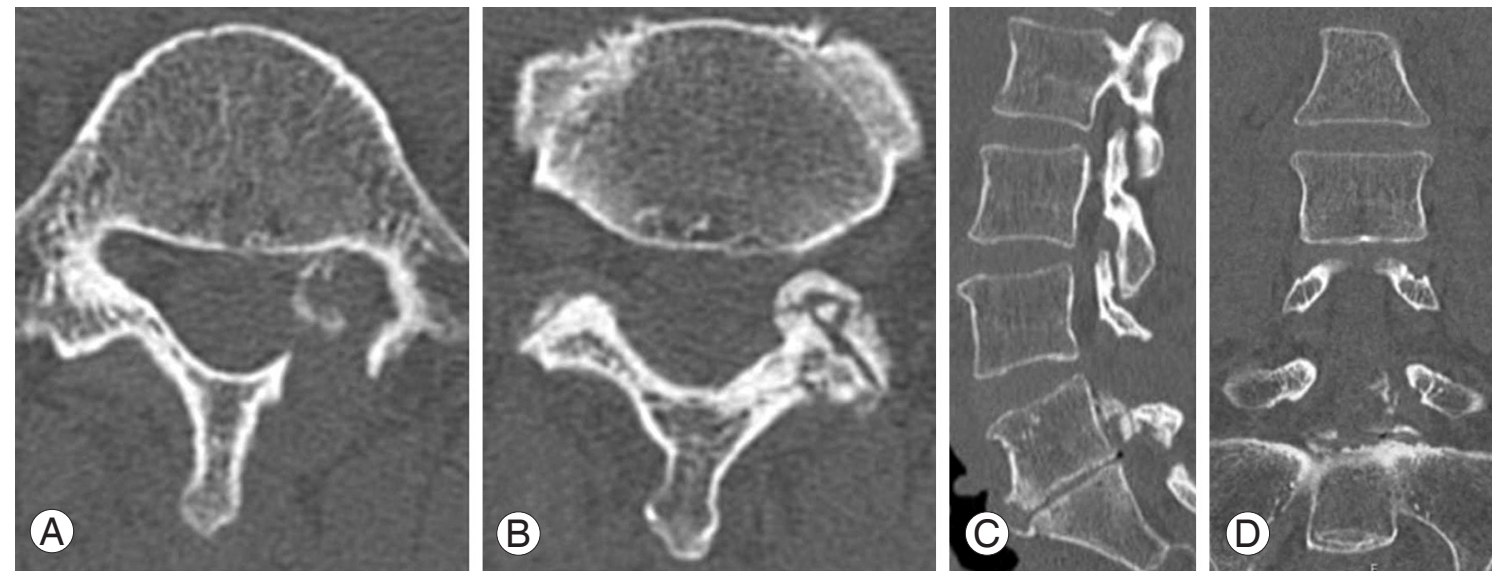

Fig. 1. (A) Axial view of the L5 showed the left L5 nerve root encircled by osteophytes. (B) Advanced osteophytic changes in the left L5/S zygapophyseal joint. (C, D) Sagittal and coronal views showed that osteophytes developed along the left L5 nerve root.
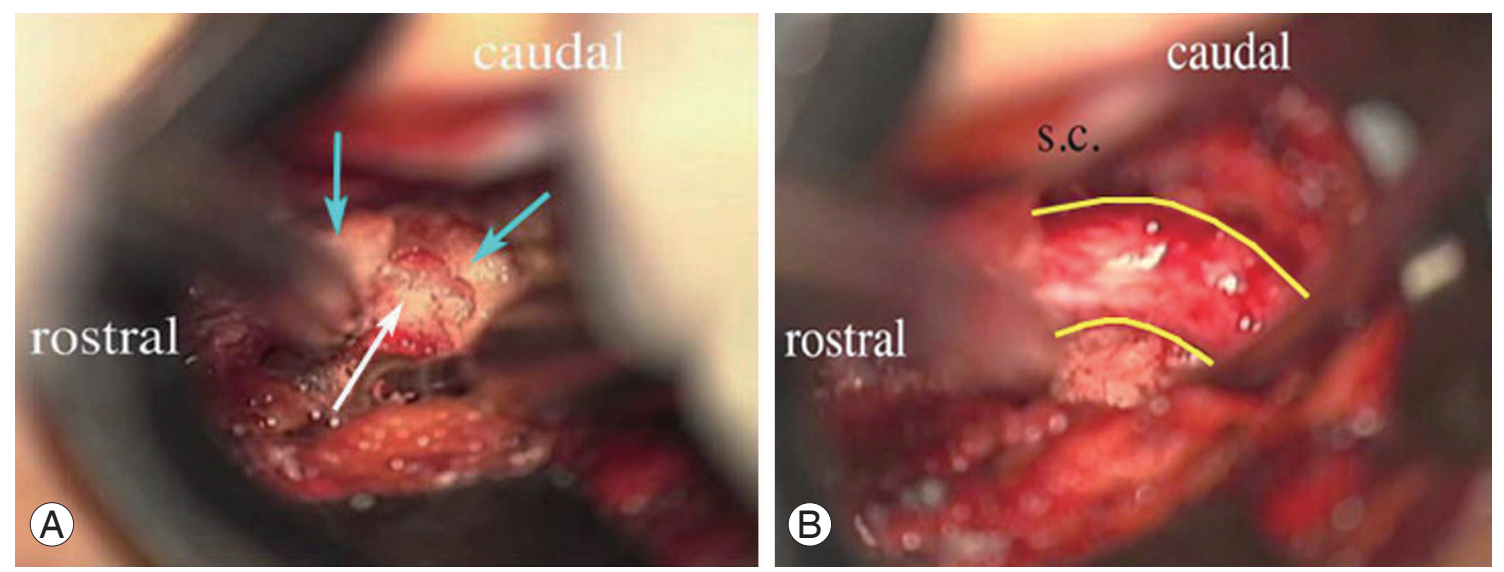

Fig. 2. (A) Operative view shows the left L5 nerve root entrapped by the ossified tissue, instead of the fat tissue in the periradicular sheath. Blue arrows indicate the ossified tissue surrounding the left L5 nerve root. White arrow indicates the left L5 nerve root. (B) The ossified tissue was removed from the nerve root. The yellow solid line indicates the boundary of the nerve root. S.C.: Spinal cord. 


\section{Discussion}

This study is a case report demonstrating a rare clinical presentation of osteophytic formation, with a specific configuration in relation to the nerve root. These advanced osteophytic changes in the lumbosacral zygapophyseal joint entrapped the L5 nerve root at the intervertebral foramen. Thus, the lumbar nerve root was compressed and sealed by the ossified tissue. In this case, the fat tissue of the epiradicular sheath disappeared after neurosurgery. The ossification of a lumbar nerve root is extremely rare, though a previous case has been reported in a child, in whom calcified tissue encased the nerve root [1].

The entrapment of lumbar nerve root can occur at three locations: the lateral recess, the intervertebral foramen and extraforamina [2-4]. Decrease in the height of the intervertebral disc, osteoarthrotic changes in the facet joints, cephalad subluxation of the superior articular process and either deflection of the ligamentum flavum or protrusion of the annulus fibrosus may constrict the intervertebral foramen sufficiently to cause entrapment of the spinal nerve root [5]. Moreover, the transforaminal ligaments [6], psuedomeningocele [7], sinovitis [8] and extraforaminal osteophytes [9] can also entrap the spinal nerve root.

Osteophytic formation in the vertebral body is a well documented phenomenon associated with degeneration and altered mechanics of the spine, both of which have been considered to be the result of aging, a physiologic response to load bearing, or intrinsic spinal disease, as etiologic factors. Osteophytes form as a specific tissue reaction to stresses and strains [10].

This patient had undergone surgery for L4-5 disc herniation in another hospital. Thus, the left L4-5 joint had already been more or less injured, before visiting our hospital. Therefore, stresses and strains on adjacent intervertebral joints had been generated after the first operation. Moreover, there were narrow lumbosacral intervertebral distances, whereas the height of L4-5 was preserved. Following the load bearing to the left L5-S zygapophyseal joint after surgery, osteophytic formation had developed.

We experienced a rare clinical presentation of osteophytic formation, with a specific configuration in relation to the nerve root. Surgeons should be aware of entrapment of the lumbar spinal nerve by advanced osteophytic changes in the zygapophyseal joint after lumbar surgery.

\section{REFERENCES}

1. Aulisa L, Pitta L, Aulisa AG, Mastantuoni G, Pola E, Leone A. Lumbar nerve root "walled" by a calcified herniated mass in a young patient. Childs Nerv Syst 2003;19:384-6.

2. Wiltse LL, Guyer RD, Spencer CW, Glenn WV, Porter IS. Alar transverse process impingement of the L5 spinal nerve: the far-out syndrome. Spine (Phila Pa 1976) 1984;9:31-41.

3. Kirkaldy-Willis JD, Heithoff KB, Tchang S. Lumbar spondylosis and stenosis. Correlation of pathological anatomy with high-resolution computed tomographic scanning. In: Post MJ, editor. Computed tomography of the spine. Baltimore: Williams \& Wilkins; 1984. p. 546-69.

4. Macnab I, McCulloch J. Disc degeneration with root irritation: the lateral zone. In: Grayson TH, editor. Backache. 2nd ed. Baltimore: Williams \& Wilkins; 1990. p. 335-79.

5. Hasegawa T, An HS, Haughton VM, Nowicki BH. Lumbar foraminal stenosis: critical heights of the intervertebral discs and foramina. A cryomicrotome study in cadavera. J Bone Joint Surg Am 1995;77:32-8.

6. Min JH, Kang SH, Lee JB, Cho TH, Suh JG. Anatomic analysis of the transforaminal ligament in the lumbar intervertebral foramen. Neurosurgery 2005;57:37-41.

7. O'Connor D, Maskery N, Griffiths WE. Pseudomeningocele nerve root entrapment after lumbar discectomy. Spine (Phila Pa 1976) 1998;23:1501-2.

8. Sampathkumar K, Rajasekhar C, Robson MJ. Pigmented villonodular synovitis of lumbar facet joint: a rare cause of nerve root entrapment. Spine (Phila Pa 1976) 2001;26:E213-5.

9. Matsumoto M, Chiba K, Nojiri K, Ishikawa M, Toyama Y, Nishikawa Y. Extraforaminal entrapment of the fifth lumbar spinal nerve by osteophytes of the lumbosacral spine: anatomic study and a report of four cases. Spine (Phila Pa 1976) 2002;27:E169-73.

10. Bick EM. Vertebral osteophytosis; pathologic basis of its roentgenology. Am J Roentgenol Radium Ther Nucl Med 1955;73:979-83. 